\title{
Formas clínicas y perfil epidemiológico de tuberculosis en niños, Hospital Nacional Dr. Mario Catarino Rivas
}

\section{Clinical forms and epidemiologic profile of tuberculosis in children Dr. Mario Catarino Rivas Hospital}

\section{Daphne Lizette Sabillón Vásquez*}

\section{RESUMEN}

Introducción. La tuberculosis (TB) es una enfermedad infecciosa de amplia distribución, cuya importancia va cada vez más en aumento. Un tercio de la población mundial está infectada y cada año ocurren en el mundo un millón de casos en menores de 15 años. En Honduras, durante el 2,008 se notificaron 2,862 casos para una incidencia de 37.1/100,000 habitantes. Objetivo. Reconocer las formas clínicas y el perfil epidemiológico de niños menores de 18 años con diagnóstico de TB. Pacientes y métodos. Se realizó un estudio descriptivo. La información fue recopilada mediante encuesta sobre características sociodemograficas, epidemiología y diagnóstico en niños con TB que fueron ingresados o manejados en consulta externa del Hospital Nacional Dr. Mario Catarino Rivas (HNMCR), durante el periodo de Julio 2013 a Julio $2014 \quad(n=56)$. Resultados. Se encontró 27 (48.2\%) niñas y 29 (51.8\%) niños con TB. En el grupo de edad de 2 a 24 meses hubo 12 (21\%) casos, 7 (12.5\%) de 3 a 6 años, 14 (25\%) de 7 a 11 años y 23 (41\%) mayores de 12 años. Las formas de la enfermedad fueron: 38 (68\%) casos de TB pulmonar y 18 (32\%) casos de TB extrapulmonar. El 18\% (10 casos) de los pacientes tiene antecedente de co-infección con VIH. Conclusiones. La TB pulmonar es la forma de presentación clínica más frecuente y afecta a escolares y adolescentes. El 22\% de casos de TB están asociados a inmunosupresión, especialmente VIH. Los principales métodos diagnósticos utilizados son: aislamiento del bacilo, la prueba de tuberculina y estudios radiológicos.

\footnotetext{
* Residente de tercer año de Pediatría, Universidad Nacional Autonoma de Honduras en el Valle de Sula UNAH-VS

* Pediatra del Centro de Atención Integral a niños del Hospital Nacional Dr. Mario Catarino Rivas HNMCR

Dirigir correspondencia a:dr_dliz@hotmail.com

Recibido: 10 de Diciembre 2014, Aprobado: 10 de Marzo 2,015
}

Luis Enrique Jovel López**

\section{PALABRAS CLAVE}

Mycobacterium tuberculosis, Tuberculosis Pulmonar, Tuberculosis, VIH.

\section{ABSTRACT}

Introduction. Tuberculosis (TB) is a widespread and increasingly important infectious disease. A third of the world-wide population is infected. Every year, one million cases occur in children under 15 years old. In Honduras, 2,862 cases were reported during 2,008 with an incidence of 37.4/100000 people. Objective. To identify the clinical forms and epidemiologic profile in children diagnosed with TB. Patients and Methods. A descriptive study was conducted. Sociodemographic, epidemiologic and diagnostic information was gathered from surveys applied to children with TB, who were admitted to or were managed at the outpatient clinic at the National Hospital Dr. Mario Catarino Rivas (HNMCR), from July 2013 to July 2014 $(n=56)$. Results. 27(48.2\%) were female and 29(51.8\%) were male. There were 12 (21\%) children between 2-24 months, 7(12.5\%) were 3-6 years old, $14(25 \%)$ cases between $7-11$ years old and $23(41 \%)$ cases were older than 12 years. The clinical forms of TB were pulmonary TB $(n=38(68 \%)$ and extrapulmonary TB $(n=18(32 \%)) .18 \%$ (10 cases) were co infected with HIV. Conclusions. Pulmonary TB is the most frequent form of TB disease affecting primarily young children and adolescents. $22 \%$ of TB cases are associated with immunodeficiency, specially TB/HIV co-infection. The main diagnostic methods used were isolation of the bacillus, tuberculin test, and radiological images.

\section{KEYWORDS}

HIV, Mycobacterium, Tuberculosis, Pulmonary Tuberculosis, Tuberculosis. 


\section{INTRODUCCIÓN}

La TB es todavía una importante causa de morbilidad y mortalidad en todo el mundo, a pesar de los grandes avances en técnicas de diagnóstico y tratamiento. Se proyecta que en los próximos 10 años la tuberculosis producirá la muerte de 30 millones de personas, afectará a 90 millones de individuos y cientos de millones se sumarán a los casi dos mil millones de personas ya infectadas. ${ }^{(1,2)}$ En los últimos años ha habido un incremento importante de casos, actualmente no sólo ataca a las naciones más sub- desarrolladas, sino también a países desarrollados (que cuentan con un mejor control de salud), teniendo una relación directa con la aparición del virus de la inmunodeficiencia humana $(\mathrm{VIH}){ }^{(3)}$

De los 8 a 12 millones de casos nuevos por año que se presentan en el mundo, cerca de 564 mil ocurren en América Latina, y de los 3 a 5 millones de muertes atribuidas a dicha enfermedad, alrededor de 250 mil ocurren en esta región. ${ }^{(3,4)}$ La TB infantil representa alrededor del 5\% de todos los casos de TB en los países desarrollados. $^{(5)}$ En los países en vías de desarrollo, 30\% de la población menor de 15 años se encuentra afectada (1,300,000 casos) y aproximadamente 450 mil mueren anualmente. Un dato preocupante es el hecho de que en la actualidad no sólo se observan más casos de TB en niños menores de 5 años, también hay un incremento de las formas extrapulmonares. ${ }^{(2)}$

En Honduras, durante el 2008 se notificaron 2862 casos para una incidencia de 37.1/ 100,000 habitantes que sitúa al país en la categoría de países con moderada incidencia. Las tendencias según las formas de $\mathrm{TB}$, reflejan la constante aunque lenta, disminución de la tasa de incidencia de la forma pulmonar a partir del 2003 y un incremento de la TB extrapulmonar probablemente secundaria a la co infección TB/VIH. La distribución etárea de casos de TB pulmonar demuestra que la población entre 15 y 34 años de edad es la más afectada (41\% de los casos). La proporción hombre: mujer es de 1.5 a 1.0, con tasas mayores en hombres a partir de los 25 años. ${ }^{(6)}$
La TB infantil está íntimamente ligada a la del adulto y se considera que los casos pediátricos son los centinelas de la comunidad. El riesgo de desarrollar la enfermedad varía en función de la edad, siendo más alta en mayores de 10 años, mientras que la presencia de formas graves predomina en menores de cinco años. ${ }^{(2,7)} \mathrm{El}$ diagnóstico de TB en niños presenta muchas dificultades. La infección suele ser reciente y la carga bacilar escasa, lo que dificulta el aislamiento del germen, tanto en esputo como en jugo gástrico. Estos factores hacen que sean menos contagiosos y, por tanto, menos peligrosos para la población, dedicándoseles menos recursos en las estrategias de control, fundamentalmente en los países más subdesarrollados. ${ }^{(8)}$

La estrategia básica para controlar la TB, es interrumpir su contagio. Para ello es fundamental el diagnóstico del enfermo bacilífero, determinar la sensibilidad del germen, iniciar lo más temprano posible el tratamiento y vigilar el cumplimiento del mismo, así como la identificación de los pacientes infectados que actúan como reservorio de la infección. . $^{(3,9-11)}$

No se cuenta con datos estadísticos sobre la situación epidemiológica actual de la TB en pediatría en la zona norte del país, particularmente áreas de afluencia del HNMCR, por lo que se decide realizar este estudio con el fin de identificar la forma de presentación clínica más frecuente de TB en niños, las características sociodemográficas de la población en estudio, comorbilidades y su efecto sobre el pronóstico del paciente, pruebas diagnósticas utilizadas y la respuesta inicial al tratamiento de primera fase.

\section{PACIENTES Y MÉTODOS}

Se realizó un estudio descriptivo, híbrido, transversal y con un componente prospectivo de seguimiento.

La población en estudio fue conformada por niños de ambos sexos, de edad comprendida entre los 2 meses a 18 años, que fueron ingresados o manejados en consulta externa del HNMCR, durante el periodo de Julio 2013 a Julio 2014, a quienes se les realizó diagnóstico 
de TB pulmonar o extrapulmonar, a través de estudios laboratoriales, inmunológicos o de imágen; o pacientes con estudios negativos o dudosos, que presentan respuesta favorable a prueba terapéutica.

Se excluyó a los pacientes que no presentaron respuesta favorable a prueba terapéutica con antifímicos, aquellos en quienes se descartó enfermedad tuberculosa, y todo paciente cuyo padre o responsable no haya autorizado su inclusión al estudio.

Para llevar a cabo esta investigación, se tomaron los principios plasmados en la Declaración de Helsinki para investigación en sujetos humanos. Se solicitó autorización a la jefatura del HNMCR y se le solicitó a cada paciente (o padre/responsable), para la obtención de datos su consentimiento informado explicándose el motivo de la investigación, haciendo hincapié en que su participación es voluntaria y asegurando la confidencialidad y anonimato de la información. En casos en los que se hizo revisión del expediente clínico, se realizó sin manipulación de los datos del mismo, con total confidencialidad.

El cuestionario constó de 20 preguntas cerradas. Fue llenado por el médico residente a cargo de la investigación, a través del interrogatorio al paciente o familiar (si está hospitalizado) o mediante revisión del expediente clínico. Los datos obtenidos fueron: datos socioeconómicos (edad, género, procedencia, nivel socioeconómico, antecedente de vacunación BCG y estado nutricional), comorbilidad asociada, contacto con algún sintomático respiratorio o enfermo tuberculoso, y se registro el método diagnóstico (clínico, epidemiológico, inmunológico, radiológico, terapéutico).

Se captaron un total de 60 niños, de los cuales se excluyer on 4 por no confirmarse el diagnóstico de tuberculosis.

Se consolidó, tabuló y analizó la información recolectada mediante el programa estadístico
Epi Info Versión 3.5.1 y Microsoft Excel 2010. Se realizó el análisis univariado y bivariado aplicando las medidas de frecuencia: porcentajes, medias y proporciones.

\section{RESULTADOS}

Al distribuirlos por edad, los casos se agruparon así: 2 a 24 meses hubo 12 (21\%) casos, 7 (12.5\%) casos de 3 a 6 años, 14 (25\%) casos de 7 a 11 años y 23 (41\%) casos mayores de 12 años.

De los 56 casos, 27 (48.2\%) de sexo femenino y $29(51.8 \%)$ masculino con una profesión. 38 (68\%) casos eran procedentes de área urbana y 18 (32\%) casos eran procedentes de área rural.

El $65 \%$ (36 casos) de los niños con diagnóstico de TB presentan algún grado de desnutrición aguda, de estos, 18 casos (32.8\%) presentan desnutrición leve, 16 casos (28.6\%) presentan desnutrición aguda moderada, y 3 casos (5.4\%) presentaron desnutrición aguda severa (gráfica No. 1). Se encontraron únicamente 10 casos de desnutrición crónica, representando el 18\% del total de casos. Solamente 8 casos (14.2\%) fueron eutróficos.

Gráfica No. 1: Distribución de niños con TB según estado nutricional.

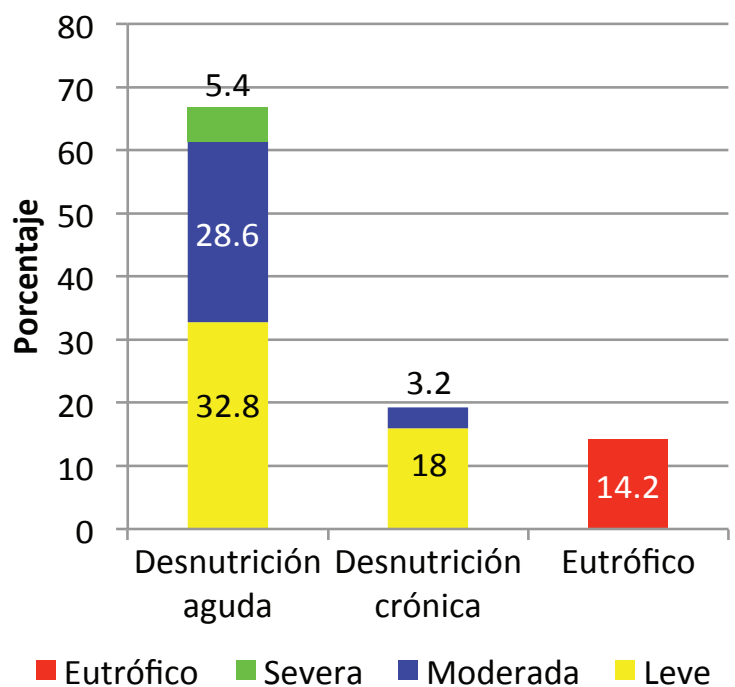

Fuente: instrumento “Formas Clínicas y Perfil Epidemiológico de niños menores de 18 años con Tuberculosis, HNMCR, Junio 2013 a Junio 2014" 
De los 56 pacientes, 45 casos (80\%) pertenecían a un estrato socioeconómico pobre y 11 casos (20\%) eran no pobres. No se encontró ningún indigente en este estudio. ${ }^{(17)}$ El $30.4 \%$ de los pacientes aseguraban vivir en hacinamiento $y$ el $69.6 \%$ lo negaron. Se encontró que solamente 2 casos (3.5\%) no habían recibido vacunación con BCG.

Al interrogar sobre la exposición a TB. El 34\% $(n=19)$ presentan antecedente de contacto con enfermo tuberculoso y/o sintomático respiratorio; el $35.7 \%(n=20)$ de los casos desconocen si hubo contacto y el $30.4 \%(n=17)$ niegan este contacto.

Las formas de la enfermedad fueron: 38 (68\%) casos con TB pulmonar y 18 (32\%) casos con TB extrapulmonar. De los 38 casos con TB pulmonar, 20 casos (36\%) se encontraron en rango de edad mayor de 12 años; 9 casos (16\%) entre los 6 a 11 años; 3 casos entre los 25 meses a 5 años; y 6 casos (11\%) entre los 2 y 24 meses de edad. Los casos de TB extrapulmonar se distribuyeron según edad así: 3 casos (5\%) en pacientes mayores de 12 años; 5 casos (9\%) entre los 25 meses a 5 años; 4 casos (7\%) entre los 6 a 11 años y 6 casos (11\%) en lactantes. (Gráfica 2).

Gráfica No. 2: Formas de TB y distribución de pacientes por edad.

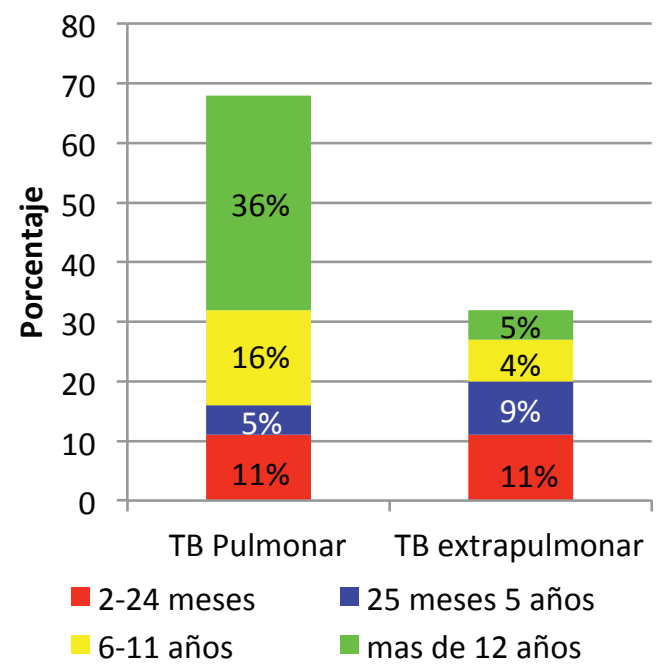

Fuente: instrumento "Formas Clínicas y Perfil Epidemiológico de niños menores de 18 años con Tuberculosis, HNMCR, Junio 2013 a Junio 2014"
En cuanto al tipo de TB pulmonar se encontró que de los 38 casos, el 10.5\% $(n=4)$ presentó TB crónica, $42 \%(n=16)$ presento TB primaria intratorácica y $44.8 \%(n=17)$ de los casos fueron TB primaria progresiva. Solamente un paciente presento TB miliar.

De los casos de TB extrapulmonar encontrados el $22.2 \%(n=4)$ presento TB pleural, $61 \%(n=11)$ fue TB ganglionar, $11 \%(n=2)$ se presentó como TB de sistema nervioso central (SNC) y se encontró un caso de pericarditis tuberculosa.

El $18 \%(n=10)$ tenían co infección con VIH de los cuales 6 son pacientes conocidos que reciben tratamiento anti retroviral (ARV), 1 no recibía $A R V$ y en 3 se realizó hallazgo incidental; 2 pacientes (3.5\%) presentaron otras comorbilidades asociadas, un paciente con linfoma Hodgkin y el segundo con leucemia linfoblástica aguda (LLA).

De los 56 casos, 3 pacientes fallecieron. Todos los pacientes que fallecieron presentaron formas severas de TB, 2 casos presentaron TB de SNC y el tercer caso presento TB miliar; en los 3 casos se encontró comorbilidad asociada, un caso asociado a co infección $\mathrm{VIH}$, otro caso asociado a LLA, y el tercer caso asociado a desnutrición severa. 2/3 de estos pacientes no tenían el antecedente de vacunación (BCG). (Tabla No. 1).

Tabla No. 1: Características de los pacientes con TB que fallecieron.

\begin{tabular}{cccc}
\hline Características & $\begin{array}{c}\text { Co- } \\
\text { Infección } \\
\text { VIH }\end{array}$ & Cáncer & $\begin{array}{c}\text { Desnutri- } \\
\text { ción Severa }\end{array}$ \\
\hline $\begin{array}{c}\text { Defunciones } \\
\text { VACUNACION } \\
\text { BCG }\end{array}$ & 1 & 1 & 1 \\
$\begin{array}{c}\text { FORMA } \\
\text { CLINICA DE }\end{array}$ & SNC & SNC & Miliar \\
TB & & & \\
EDAD & $\begin{array}{c}6 \text { a } 11 \\
\text { años }\end{array}$ & $\begin{array}{c}6 \text { a 11 } \\
\text { años }\end{array}$ & $\begin{array}{c}25 \text { meses a } \\
5 \text { a años }\end{array}$ \\
\hline
\end{tabular}

Fuente: instrumento "Formas Clínicas y Perfil Epidemiológico de niños menores de 18 años con Tuberculosis, HNMCR, Junio 2013 a Junio 2014" 
De los métodos diagnósticos, la radiología orientó el diagnóstico en un $84 \%{ }^{(47)}$ de los casos un $76 \%{ }^{(43)}$ de los casos presentaron una prueba de tuberculina (PPD) positiva y en $64 \%{ }^{(36)}$ de los casos se logró aislar el bacilo. Se confirmó el diagnóstico a través de cultivo del bacilo y/o hallazgo histopatológico a través de biopsia en el $18 \%{ }^{\left({ }^{10)}\right.}$ de los casos (gráfica No. 3).

Gráfico No. 3: Número de casos detectados según método diagnóstico.

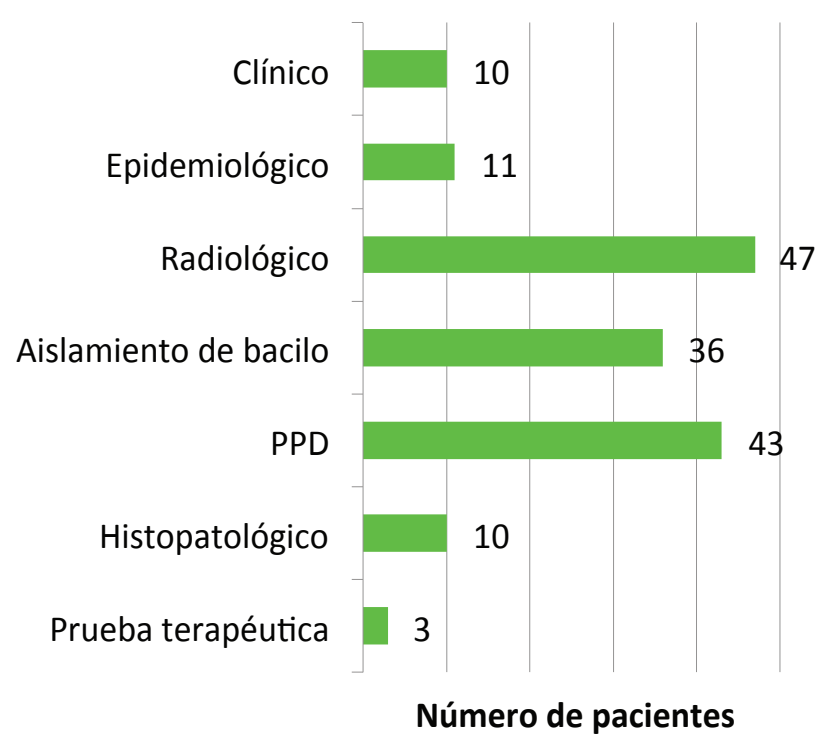

Fuente: instrumento "Formas Clínicas y Perfil Epidemiológico de niños menores de 18 años con Tuberculosis HNMCR, Junio 2013 a Junio 2014"

El $5(9 \%)$ de los pacientes presentaron respuesta no satisfactoria al iniciar el tratamiento antifímico de primera fase, dos de estos abandonaron tratamiento previamente y se detectaron en esta ocasión como recaída; los 3 pacientes restantes fallecieron.

\section{DISCUSIÓN}

Honduras continúa teniendo problemas con esta enfermedad, de manera silenciosa y poco visible. En los medios de comunicación se percibe más el problema de la violencia, el SIDA y el dengue, permaneciendo oculto el de la TB. Sin embargo, la TB, lenta y de manera oculta afecta cada día más a la población, en particular por su asociación con la infección por $\mathrm{VIH}$, y la aparición de formas resistentes, que se transmiten y diseminan entre comunidades, regiones y países. ${ }^{(6)}$

Las acciones de los programas de control de tuberculosis como problema de salud pública se han dirigido generalmente a los adultos; el abordaje de la enfermedad en menores de 15 años se dificulta por la falta de conocimiento claro del problema y a los inconvenientes en el diagnóstico. Debido a la poca contagiosidad de estos casos, es normal que el Programa Nacional de Control de Tuberculosis se concentre en la identificación de las fuentes de contagio, es decir, los adultos bacilíferos que mantienen la cadena de transmisión. ${ }^{12,13)}$

En este estudio el $66 \%$ de los casos los pacientes se encontraban en edades comprendidas de 6 a 18 años, lo cual concuerda con datos estadísticos internacionales en los cuales se reporta que el riesgo de desarrollar la enfermedad varía en función de la edad, siendo más alta en mayores de 10 años, mientras que la presencia de formas graves predomina en menores de cinco años. ${ }^{(2,7)}$

La distribución de los casos por sexo no permite considerar que existan factores de riesgo asociados a la presencia de la enfermedad.

La mayoría de los casos (68\%) detectados provienen de área urbana. De estos un $70 \%$ viven en el departamento de cortes, probablemente por tener mejor acceso a servicios de salud; en el área rural, se da un alto índice de sub registro asociado a falta de personal de salud capacitado para sospechar y detectar muchos casos que no tienen presentación clínica típica sobre todo en niños en los cuales se presentan formas de TB extrapulmonares, estos niños reciben manejos inadecuados y favoreciendo a complicaciones como la muerte, al no haber recibido atención médica en un centro hospitalario. Y así estos casos no son reportados. 
La mayoría de los pacientes presentaron un estado nutricional deficiente. Reviste una importancia especial el aspecto nutricional, puesto que los niños desnutridos tienen mayor riesgo de padecer enfermedades contagiosas; así lo muestran algunos estudios, en donde los casos más graves los presentaron niños de menor peso. ${ }^{(2,7)}$

El $80 \%$ de los pacientes pertenecen a un estrato social pobre. El mapa de distribución de la tuberculosis coincide con el de la pobreza. ${ }^{(1,7)}$ El mayor riesgo de infección se encuentra entre los contactos próximos al paciente bacilífero, especialmente aquellos que comparten la vivienda, lo que se agrava cuando existe hacinamiento y falta de ventilación. En estos casos, las tasas de infección pueden llegar al 60\%.(2) Contradictoriamente, solo el $30 \%$ de los casos refieren vivir en hacinamiento.

El diagnóstico de un caso de tuberculosis infantil, representa un evento centinela dentro de una comunidad, pues sugiere una transmisión reciente por parte de un adulto bacilífero. Por lo que un diagnóstico temprano es sumamente importante, tanto para el control en una población, como para el inicio de un tratamiento eficaz para su erradicación. ${ }^{(8,14,15)}$ En este estudio la mayor parte de los casos negaban o desconocían si hubo contacto con una persona sintomática respiratoria, dato alarmante ya que no fue posible identificar la fuente infectante en la mayoría de los casos.

El riesgo de desarrollar la enfermedad varía en función de la edad, siendo más alta en mayores de 10 años, mientras que la presencia de formas graves predomina en menores de cinco años. El $85 \%$ de los casos de TB se presenta como TB pulmonar, solamente un 15 a $20 \%$ de los casos se presentan como TB extrapulmonar. ${ }^{(2)}$ En este estudio el $68 \%$ de los casos presenta tuberculosis pulmonar y afecta a escolares y adolescentes principalmente; los casos de TB extrapulmonar predominan en niños menores.

En el 2008 se reportó una incidencia de co- infección VIH del 17\%.(6) En este estudio se encontró que el $18 \%$ de los pacientes tiene antecedente de co infección con VIH. Aunque se reporte a nivel internacional que el $\mathrm{VIH}$ ha permitido un incremento en el número de casos de TB, en los niños este fenómeno no está estudiado, pero es muy probable que la infección por el VIH se haya acompañado también de un incremento de los casos de tuberculosis infantil.

La co infección de M. tuberculosis y $\mathrm{VIH}$ resulta en un rápido deterioro de la función inmune, replicación viral y progresión de la enfermedad por VIH y, muy frecuentemente, en otras infecciones severas. En este tipo de pacientes, la tuberculosis presenta signos y síntomas muy inespecíficos, lo que hace muy difícil su diagnóstico. ${ }^{(16)}$

La tuberculosis cavitaria, la diseminación extrapulmonar y la miliar ocurren más frecuentemente en niños infectados con VIH. La respuesta al tratamiento antituberculoso estándar es muy bajo en niños infectados con VIH en comparación con los no infectados y con picos muy altos de mortalidad. $(2,13,16)$

Todos los pacientes que fallecieron presentaron formas severas de TB y comorbilidad asociada; $2 / 3$ no tenían el antecedente de vacunación (BCG). Cuando la tuberculosis pulmonar se desarrolla en un niño infectado por el $\mathrm{VIH}$, las características tienden a ser típicas de la TB infantil de un paciente inmunocompetente pero la enfermedad a menudo progresa más rápidamente y las manifestaciones clínicas son más severas. De igual manera, hay una tendencia incrementada al desarrollo de tuberculosis extrapulmonar o de formas severas de TB como la miliar, incrementando la mortalidad. ${ }^{(13)}$ De los tres pacientes que fallecieron, dos presentaron TB extrapulmonar. En pediatría la curación ocurre en el 95\% de los niños inmunocompetentes afectados; sin embargo, existe el riesgo de reactivaciones en alguna etapa de la vida. El 5\% restante evoluciona a enfermedad pulmonar o dise- 
minada, sobre todo en menores de 2 años, quienes tienen mayor riesgo de presentar formas graves o extrapulmonares, incrementando el riesgo de mortalidad. ${ }^{(2)}$ Un meta-análisis reciente ha demostrado que la BCG tiene una efectividad del $73 \%$ frente a la meningitis tuberculosa y del $77 \%$ frente a la tuberculosis miliar. En otro estudio similar, la efectividad frente a cualquier forma de tuberculosis fue alrededor del 50\% mientras que para la tuberculosis diseminada fue casi del $80 \%$. Se ha estimado que la duración de la protección de la BCG se extiende hasta 15 años. ${ }^{(3,5)}$

Los métodos diagnósticos utilizados fueron el aislamiento del bacilo en muestras de esputo o jugo gástrico, la PPD y los estudios radiológicos (rayos x y TAC). En la literatura se reporta que en niños el aislamiento del bacilo mediante aspirado nasotraqueal y lavado gástrico han sido comparadas entre sí, y su sensibilidad y especificidad varían, siendo el primero más sensible (62\%) y específico (98\%) para TB pulmonar. La PPD es otro método básico que suele ser positivo en el $86,7 \%$ de los casos. ${ }^{(17)}$

El $90 \%$ de los pacientes presentan una respuesta favorable y buena tolerancia al tratamiento de primera fase; se describe en la literatura que la respuesta al tratamiento antituberculoso estándar es muy inadecuada en niños infectados con VIH en comparación con los no infectados y tiene picos muy altos de mortalidad.(18)

El país requiere una guía de manejo de los casos de tuberculosis en menores de 15 años enfocada en las actividades de detección, diagnóstico, tratamiento, seguimiento y prevención de farmacorresistencia, que pueda ser aplicada por médicos generales y especialistas en todo el territorio nacional. Se recomienda a la Secretaria de Salud, fortalecer la capacitación de personal y su distribución a las áreas de difícil acceso a servicios de salud, para lograr adecuada detección y diagnóstico oportuno de ésta enfermedad que todavía tiene un impacto importante sobre nuestra población en general, y particularmente población pediátrica en quienes se manifiestan los casos más graves.

Además, se debe garantizar la disponibilidad de pruebas básicas para el diagnóstico de TB como ser la PPD y medios de cultivo para el bacilo en centros hospitalarios como el HNMCR, y así poder ofrecer la posibilidad de hacer un diagnóstico rápido y oportuno. No se debe olvidar también la importancia del abastecimiento continuo de los fármacos para cumplir el tratamiento de primera fase para TB, ya que este desabastecimiento favorece la resistencia farmacológica, el retraso de inicio de tratamiento y aumento del riesgo de contagio.

\section{AGRADECIMIENTOS}

A todos los responsables del departamento de Epidemiología del Hospital Dr. Mario Catarino Rivas, por el suministro de la información de los reportes semestrales.

A Dr. Carlos Fuentes, asesor metodológico.

\section{CONFLICTO DE INTERESES}

Los autores del artículo hacen constar que no existe, de manera directa o indirecta, ningún tipo de conflicto de intereses financieros, académicos o personales que puedan poner en peligro la validéz de lo comunicado. 


\section{BIBLIOGRAFÍA}

1. Global Tuberculosis Report 2013. Geneva, World Health Organization, [Citado 2014, noviembre 27] 2013. (WHO/HTM/TB/2013). Disponible en: http:// apps.who.int/iris/bitstream/10665/91355/1/9789241564656 _eng.pdf

2. Orozco I, Nesbitt C, González S. Tuberculosis en pediatría: epidemiología. Revista de Enfermedades Infecciosas en Pediatría. [Citado 2014, noviembre 27] 2009; XXII (87):83-90 Disponible en:

http://www.medigraphic.com/pdfs/revenfinfped/eip-2009/eip091e.pdf

3. Velasco Bernardo RM. Actualización en tuberculosis. REV PATOL RESPIR [Citado 2014, noviembre 28] 2009; 12(SUPL.1):30-33. Disponible en: http://www.revistadepatologiarespiratoria.org/descargas/pr_12-s1_30-33.pdf

4. Grupo de trabajo de tuberculosis de la Sociedad Española de Infectología Pediátrica. Documento de consenso sobre el tratamiento de la exposición a tuberculosis y de la infección tuberculosa latente en los niños. An Esp Pediatr (Barc). [Citado 2014, noviembre 28] 2006; 64: 59-65. Disponible en: http://analesdepediatria.org/es/documento-consenso-sobre-el-tratamiento/ articulo/ 13083834/

5. Ruiz Contreras. Mesa Redonda: Actualización del abordaje de la tuberculosis en Pediatría. Bol Pediatr. [Citado, 2014 noviembre 29] 2011; 51: 234-240. Disponible en: http://www.sccalp.org/documents/0000/ 1789/BolPediatr2011_51_225_229.pdf

6. Varela C. Plan estratégico nacional para el control de la tuberculosis. PENTB 20092015. Resumen. Rev. Med. Hondur. 2010;78 (1):39-48.

7. Programa de Apoyo a la Reforma de Salu-
d/PARS • Ministerio de la Protección Social. Guía de atención de la tuberculosis pulmonar y extrapulmonar. Guías de promoción de la salud y prevención de enfermedades en la salud pública. Colombia. 2005.

Disponible en:

http://www.minsalud.gov.co/Documetos $\% 20 y \% 20$ Publicaciones/GUIAS\%20 DE\%20ATENCION\%20-TOMO\%20DOS.pdf

8. Stockdale AJ, Duke T, Graham S, Kelly J. Evidence behind the WHO guidelines:

hospital care for children: what is the diagnostic accuracy of gastric aspiration for the diagnosis of tuberculosis in children? J Trop Pediatr. [Citado 2014, noviembre 29] 2010; 56(5):291-298. Disponible en: http:// www.ncbi.nlm.nih.gov/pubmed/20 817689/

9. Comité Nacional de Infectología y Neumología. Tuberculosis Infantil. Modificaciones a los criterios de diagnóstico y tratamiento de la tuberculosis infantil. Arch. Argent. Pediatr. [Citado 2014, noviembre 30] 2007; 105(1): 54-5. Disponible en:

http://www2.sap.org.ar/docs/publicaciones/archivosarg/2007/159.pdf

10. Mejía O. Tuberculosis Infantil: Un enfoque actual. Rev Med Hondur [Citado 2014, noviembre 30] 2007;75(1):30-42. Disponible en: http://www.bvs.hn/RMH/pdf/2007/ pdf/Vol75-1-2007-8.pdf

11. Cuevas LE, Browning R, Bossuyt $P$, Casenghi M, Cotton MF. Evaluation of Tuberculosis Diagnostics in Children: 2. Methodological Issues for Conducting and Reporting Research Evaluations of Tuberculosis Diagnostics for Intrathoracic Tuberculosis in Children. Consensus From an Expert Panel. The Journal of Infectious Diseases. [Citado 2014, noviembre 27] 2012; 205(suppl 2) S209-215. Disponible en:

http://jid.oxfordjournals.org/content/205/suppl_2/S209.full.pdf 
12. Mejia O. Presentación clínica y radiológica de la tuberculosis pediátrica. Rev. Med. Hondur. [Citado 2014, noviembre 30] 2010; 78(1): 29-32. Disponible en: http://www.bvs.hn/RMH/pdf/2010/pdf/Vol78-1-201010.pdf

13. Cruz Anleua ID, Velásquez Serratos JR. Tuberculosis infantil. ¿Cómo diagnosticarla? Arch. Argent. Pediatr. [Citado 2014, noviembre 29] 2012; 110(2):144-151. Disponible en:

http://www.scielo.org.ar/pdf/aap/v110n2/v110n2a11.pdf

14. Newton SM, Brent AJ, Anderson S, Whittaker E, Kampmann B. Paediatric tuberculosis. Lancet Infect Dis. [Citado, 2014, noviembre 29] 2008; 8(8):498-510. Disponible en: http://www.ncbi.nlm.nih.gov/pubmed/ 18652996

15. Sociedad Argentina de Pediatría, Comité Nacional de Neumonología, Comité Nacional de Infectología. Criterios de diagnóstico y tratamiento de la tuberculosis infantil. Arch.argent.pediatr. [Citado 2014, noviembre 30] 2002; 100(2).
Disponible en:

http://www.sap.org.ar/docs/profesionales/consensos/159(1).pdf

16. Pediatric Tuberculosis Collaborative Group. Targeted Tuberculin skin testing and treatment of latent Tuberculosis Infection in children and adolescents. Pediatric. [Citado 2014, diciembre 1] 2004; 114: 1175-201. Disponible en:

http://pediatrics.aappublications.org/content/114/Supplement_4/1175.full.pdf

17. Treatment of tuberculosis Guidelines, 4th ed. Geneva, World Health Organization, 2010. (WHO/HTM/TB/2009.420).

Disponible en: http://www.ncbi.nlm.nih.gov/books/NBK138748/pdf/TOC.pdf

18. Organización Mundial de la Salud. Cuestiones prioritarias en materia de investigación sobre la tuberculosis y la infección por el $\mathrm{VIH}$ en entornos de recursos limitados donde la infección por el VIH es prevalente. 2010. (WHO/HTM/TB/2010.8) Disponible en: http://whqlibdoc.who.int/publications/ 2011/9789243500300_spa.pdf 